POS $\quad$ PROCEEDINGS

\title{
Conformal finite size scaling of twelve fermion flavors
}

\author{
Zoltán Fodor \\ Department of Physics, University of Wuppertal \\ Gaußstrasse 20, D-42119, Germany \\ Jülich Supercomputing Center, Forschungszentrum \\ Jülich, D-52425 Jülich, Germany \\ Email: fodorabodri.elte.hu
}

\section{Kieran Holland}

Albert Einstein Center for Fundamental Physics, Institute for Theoretical Physics,

Bern University, Sidlerstrasse 5, CH-3012 Bern, Switzerland

Department of Physics, University of the Pacific, 3601 Pacific Ave, Stockton CA 95211, USA

Email: khollandepacific.edu

\section{Julius Kuti}

Department of Physics 0319, University of California, San Diego

9500 Gilman Drive, La Jolla, CA 92093, USA

E-mail: jkuti@ucsd.edu

\section{Dániel Nógrádi}

Institute for Theoretical Physics, Eötvös University

H-1117 Budapest, Hungary

Email: nogradi@bodri.elte.hu

\section{Chris Schroeder}

Physical Sciences Directorate, Lawrence Livermore National Laboratory

Livermore, California 94550, USA

E-mail: schroeder10@llnl.gov

\section{Chik Him Wong*}

Department of Physics 0319, University of California, San Diego

9500 Gilman Drive, La Jolla, CA 92093, USA

E-mail: rickywong@physics.ucsd.edu

Extended simulation results and their analysis are reported in a strongly coupled gauge theory with twelve fermion flavors in the fundamental SU(3) color representation. The conformality of the model is probed using mass deformed conformal finite size scaling (FSS) theory driven by the fermion mass anomalous dimension. Two independent conformal FSS fitting procedures are used in the analysis. The first one deploys physics motivated scaling functions, complemented by a second fitting procedure with spline based general B-forms for the scaling functions. The results at fixed gauge coupling show unresolved problems with the conformal hypothesis.

The 30 International Symposium on Lattice Field Theory - Lattice 2012,

June 24-29, 2012, Cairns, Australia

\footnotetext{
*Speaker.
} 


\section{Introduction}

The frequently discussed model of twelve fermions in the fundamental representation of the $\mathrm{SU}(3)$ color gauge group remains controversial with continuing recent efforts from several lattice investigations [1-13] where more extended references can be found for the earlier history of the model. The focus of these lattice studies is to establish conformality or chiral symmetry breaking in the bulk phase close to the continuum limit. At finite cutoff, two different strategies can be used to deal with finite volume dependence while probing the conformal and $\chi$ SB hypotheses of the bulk phase. The first strategy extrapolates the spectrum to infinite volume at fixed fermion mass $m$ where the leading finite size correction is exponentially small and assumed to be determined by the lowest mass with pion quantum numbers. From the mass spectrum of the infinite volume extrapolation the mass deformed conformal scaling behavior can be probed and compared with $\chi \mathrm{SB}$ behavior when the fermion mass is varied in the infinite volume limit. This limit requires very large volumes and it is difficult to reach with existing computational resources. Probing the conformal hypothesis without intrinsic scale, the second strategy takes full advantage of the conformal FSS behavior when approaching the $m=0$ critical surface at fixed finite size $L$. Different from the first strategy, the finite volume corrections are not exponentially small and a much larger data set can be analyzed closer to the critical surface. Results from the conformal FSS analysis are presented here, significantly extending what was reported earlier [1].

\section{The algorithm and the data base from the simulations}

We have used the tree-level Symanzik-improved gauge action with $\beta=2.2$ gauge coupling and with two levels of stout smearing in the staggered fermion action [14]. The standard HMC algorithm was used with multiple time scales and the Omelyan integrator. Our error analysis of hadron masses used correlated fitting with double jackknife procedure on the covariance matrices. The time histories of the fermion condensate, the plaquette, and correlators are used to monitor autocorrelation times in the simulations.

Extending earlier work [1], we have new simulation results at $\beta=2.2$ in the fermion mass range $\mathrm{m}=0.002-0.025$ at lattice volumes $20^{3} \times 40,24^{3} \times 48,28^{3} \times 56,32^{3} \times 64,40^{3} \times 80$, and $48^{3} \times 96$. The extended data base now has the $\mathrm{m}=0.002-0.035$ fermion mass range with a span close to a factor of twenty. The new low fermion mass range $\mathrm{m}=0.002,0.004,0.006,0.008$ is used in the conformal FSS analysis which over the full set extends the lattice pion correlation length from 2.5 to 20 in the infinite volume limit. Results from the two lowest masses at $\mathrm{m}=0.002,0.004$ are not included in the current analysis and will be reported later. For further control on finite volume dependence, large $48^{3} \times 96$ runs were continued to two thousand trajectories at $\mathrm{m}=0.01$ and $\mathrm{m}=0.015$. Four runs were further added at $40^{3} \times 80$ with $\mathrm{m}=0.01,0.15,0.02,0.025$. The new and updated data sets were subjected to conformal FSS analysis and $\chi$ SB tests of the $\langle\bar{\psi} \psi\rangle$ chiral condensate. Preliminary analysis of the results already appeared a few months ago [2].

\section{The bulk phase diagram}

The bulk phase structure of the model remains controversial, particularly the critically important weak coupling phase. In addition to our spectroscopy and conformal FSS runs, we ran 
extensive scans at various fixed volumes and fixed fermion masses to explore the bulk phase structure. The bare coupling $\beta$ was varied over a large range starting from very small $\beta$ values deep in the strong coupling regime to the weak coupling phase at $\beta=2.2$ where the conformal and $\chi \mathrm{SB}$ analyses were done. Fermion masses $\mathrm{m}=0.007,0.01,0.02$ were used in the scans with spatial lattice sizes $L=8,12,16,20,24,32$ running a large densely spaced set in the important and much discussed intermediate region in transit from strong coupling to weak coupling. These scans were also extended to $N_{f}=2,4,6,8,10,12,14,16$ flavors. Two representative scans of the bulk behavior
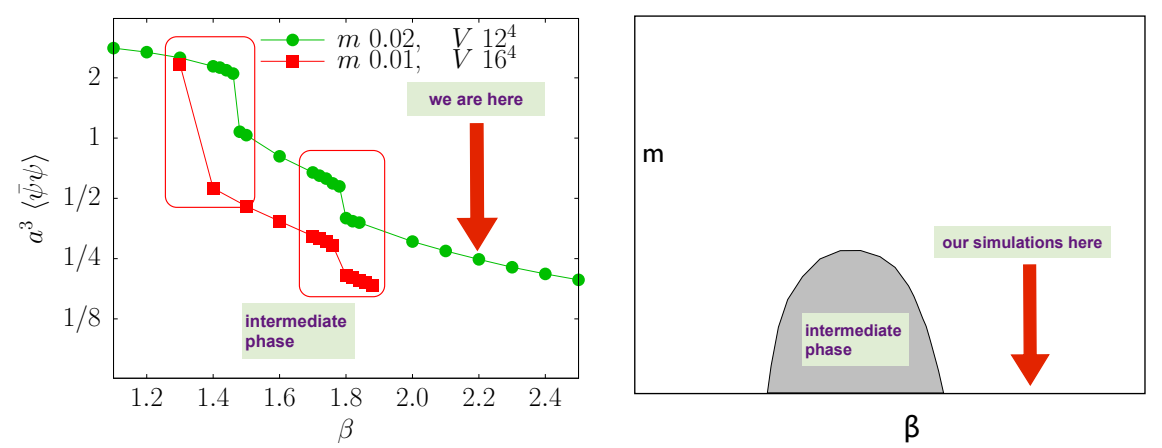

Figure 1: On the left, scans of the phase diagram by monitoring the chiral condensate are plotted as a function of $\beta$ at two different fermion masses for $N_{f}=12$. The schematic bulk phase diagram is sketched on the right, a plot suggested from findings in [9].

of the chiral condensate $\langle\bar{\psi} \psi\rangle$ are shown in Figure 1 as we vary $\beta$ from strong to weak coupling. Three distinct regions emerge at fixed volume and fixed fermion mass showing strong coupling behavior for $\beta<1.4$ with a large chiral condensate, an intermediate phase for $1.4<\beta<1.8$ with a sudden drop in $\langle\bar{\psi} \psi\rangle$, and a weak coupling phase for $\beta>1.8$ with a further drop in $\langle\bar{\psi} \psi\rangle$. We also observed another distinct feature of the intermediate phase (first seen in [9]) where the pion correlator exhibits oscillations associated with the appearance of a non-degenerate parity partner state. This structure does not exist in the weak coupling phase. A similar pattern of phases was also seen in scans at $N_{f}=8$. Our physics simulations and conformal FSS hypothesis tests were done well inside the weak coupling phase at $\beta=2.2$ without influence from the intermediate phase, as indicated in Figure 1.

A similar phase structure has been observed independently by three different groups $[4,9,12]$. The newfound order parameter of broken shift symmetry in the intermediate phase is an interesting additional development in the study of the esoteric intermediate phase when HYP smearing is used in the staggered fermion action [9]. The intermediate phase only exists in a finite interval of the lattice gauge coupling for small enough fermion masses, as schematically sketched in Figure 1. The real interest is, of course, in the nature of the weak coupling phase. Based on axial U(1) symmetry considerations, arguments were presented in [12] in favor of conformal symmetry in the weak coupling phase. This argument was criticized in [9] based on new details of the broken shift symmetry with chiral symmetry restoration they discovered at zero temperature in the bulk intermediate phase.

Weak coupling results on the Polyakov loop, the chiral condensate, and spectroscopy were also presented in [9] as indications of conformal symmetry in the weak coupling phase. The blocked Polyakov loop was reported to jump from zero to a large finite value in crossing to the weak 
coupling phase [9]. A confining potential was reported in the intermediate phase with broken shift symmetry which turned into a Coulomb potential without a string tension in the weak coupling phase [9]. It was also asserted that the observed chiral condensate and the related Dirac spectrum show the recovery of exact chiral symmetry in the massless fermion limit of the weak coupling phase consistent with observed degeneracies of parity partners even at finite fermion masses. In conclusion, conformal behavior was suggested for the bulk weak coupling phase.

The results reported in [9] suggesting a chirally symmetric deconfined conformal phase are in disagreement with what we found earlier [1] and in the extended new analysis [2,3]. Using lattice volumes several times larger than the simulations in [9] we find a vanishing Polyakov loop at zero temperature in the weak coupling phase and a confining potential at a pion correlation length which is significantly larger and consequently more relevant than the related findings in [9]. We also observe the splitting of parity partners at finite fermion mass. Consequently, and differing from [9], our findings in large volumes are consistent with a chirally broken weak coupling phase. We turn now to conformal FSS tests of the conformal hypothesis in the weak coupling phase gathering further evidence towards more definitive conclusions.

\section{Conformal finite size scaling analysis}

The expected leading FSS form for any mass $M$ in the spectrum, or for the decay constant $F_{\pi}$, scaled with the linear size $L$ of the spatial volume, is given by a scaling function $L \cdot M=f(x)$ where $x=L \cdot m^{1 / 1+\gamma}$ is the conformal scaling variable. The scaling form sets in close to the critical surface for small $m$ values. The scaling functions $f(x)$ can depend on the quantum numbers of the states but the scaling variable is expected to have the same form with identical $\gamma$ exponent in each quantum number channel [15-19]. In sub-leading order there are conformal FSS scaling violation effects which are exhibited as a combined cutoff and $L$-dependent leading correction with the modified form $L \cdot M=f(x)+L^{-\omega} g(x)$ where the scaling correction exponent $\omega$ is determined at the infrared fixed point (IRFP) $g^{*}$ of the $\beta$-function as $\omega=\beta^{\prime}\left(g^{*}\right)$. This assumes that the mass deformation away from the critical surface is the only relevant perturbation around the IRFP. The leading scaling correction term close enough to the critical surface dominates any other corrections which are further supressed by additional inverse powers of $L$. To detect the leading scaling violation effect requires high precision data with fits to scaling functions $f(x)$ and $g(x)$ and the critical exponent $\omega$.

\subsection{Conformal FSS fitting procedure with restricted scaling functions}

We applied conformal FSS theory to our data sets in the fermion mass range $m=0.006-0.035$ with lattice sizes ranging in the fits from $20^{3} \times 40$ to $48^{3} \times 96$. Two different FSS fitting procedures were applied. In the first procedure, we defined a scaling function $f(x)$ for each mass $\mathrm{M}$ with five independent fitting parameters. The fitting function $f(x)$ was divided into two regions separated at the joint $x=x_{\text {cut }}$. Different forms were chosen on the two sides of $x_{\text {cut }}$ for the expected conformal behavior. For large $x>x_{\text {cut }}$, the function $f(x)=c_{1} x+c_{\text {exp }}\left(c_{1} x\right)^{-1 / 2} \exp \left(-c_{1} x\right)$ with parameters $c_{1}$ and $c_{\text {exp }}$ describes the $L$-independent limit $M \sim c_{1} m^{1 / 1+\gamma}$ at fixed $m$ and $L \rightarrow \infty$. The $c_{\text {exp }}$ amplitude sets the size of the leading small exponential correction from the finite volume effect of the lightest Goldstone pion state wrapping around the spatial volume. Since $f(0)=c_{0}$ is expected 

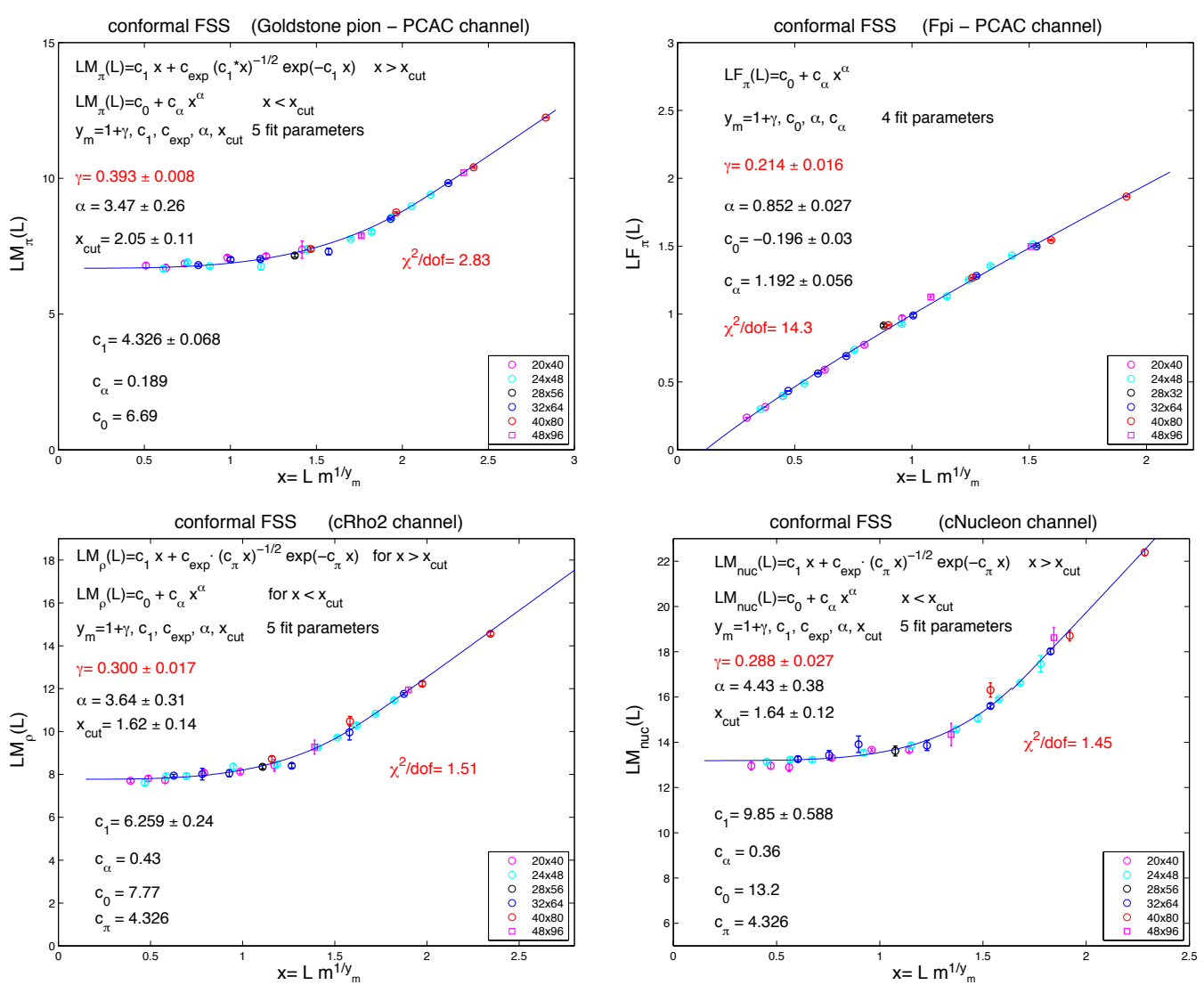

Figure 2: Conformal FSS fits in four different quantum number channels. The fits are performed in each channel separately. Since the $\gamma$ values vary considerably from channel to channel, a simultaneous global fit to the combined channels with the same $\gamma$ exponent, as required by conformal FSS theory, is bound to fail.

from conformal FSS with some power corrections at small $x$, we applied the simple ansatz $f(x)=$ $c_{0}+c_{\alpha} x^{\alpha}$ for $x<x_{\text {cut }}$ (a more general polynomial function in the small $x$ region will not change the conclusions from the fits). From the fit to the PCAC Goldstone pion channel the parameter $c_{\pi}=c_{1}$ was determined and used as input in the exponential terms of the other channels with $\exp \left(-\mathrm{c}_{\pi} \mathrm{L}\right)$. The critical exponent $\gamma$ was included among the five fitting parameters, in addition to $c_{0}, c_{1}, c_{\text {exp }}$, and $x_{\text {cut }}$.

The composite particle masses in several quantum number channels can be reasonably fitted with conformal scaling functions $f(x)$ as shown in Figure 2 but the values of the critical exponent $\gamma$ are incompatible across different channels. The required global conformal FSS fit will fail with a single exponent $\gamma$ across all quantum numbers. In the fits for $F_{\pi}$ in the PCAC pion channel we only kept four parameters because the asymptotic form with exponentially small correction was zero within error. Actually, the data of $F_{\pi}$ did not allow a successful conformal fit with any shape chosen for its scaling function $f(x)$ which looks very different from the scaling functions of composite particle masses. The unexpectedly curious behavior of the $F_{\pi}$ data set against conformal FSS remains unresolved. Our dataset is not accurate enough to successfully resolve subleading corrections in the fits. 


\subsection{Generalized FSS fitting procedure with spline based general B-form}

Following a new fitting strategy, we investigated if the failed global conformal FSS analysis can be attributed to restrictions on the conformal scaling functions $f(x)$. The restrictions were manifest in the physics-motivated fitting procedure we applied above. Our new general approach is different from [20,21] but addresses related issues. We developed a general least-squares fitting procedure to the scaling functions using the B-form of spline functions [22] without additional restrictions. In this procedure, the function $f(x)$ is described by piece-wise polynomial forms constructed from spline base functions with general coefficients in overlapping intervals of the scaling variable $x$ which depends on the exponent $\gamma$. The shape of the B-form can be changed without limitations by increasing the number of base functions and the number of scaling intervals in $x$ bracketing the overlapping data range. The details of this new analysis will be reported elsewhere.
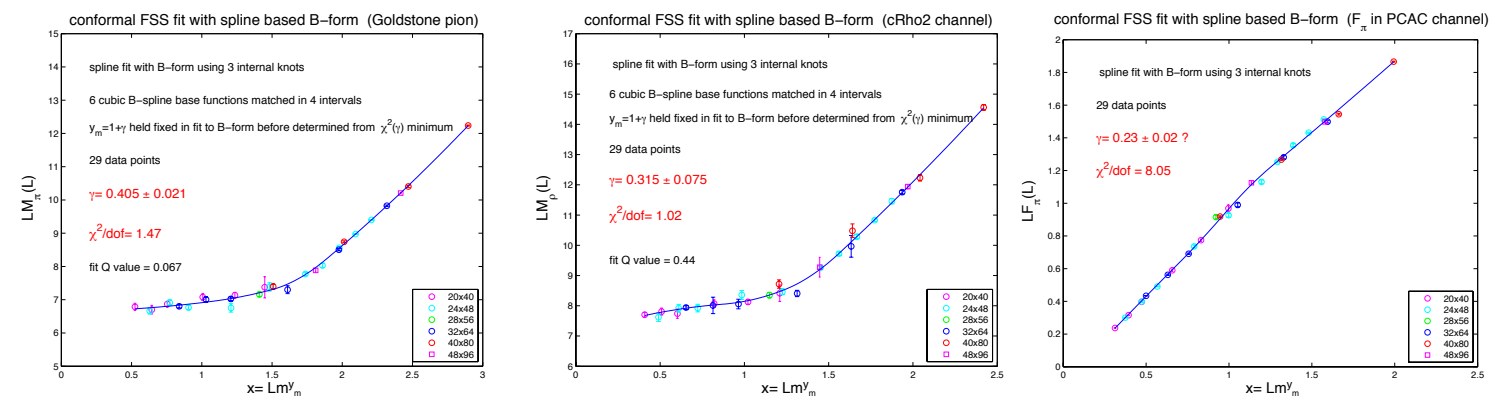

Figure 3: Conformal FSS fits using spline based B-forms in three different channels. The fits are performed in each channel separately with the question mark on $\gamma$ indicating difficulties of error estimates in bad fits of $F_{\pi}$.

Our fitting procedure in its setup requires two steps. In the first step, for any fixed choice of the exponent $\gamma$, the best fitted function $f(x)$ is determined in spline function B-form from the minimization of the weighted $\chi^{2}$ expression. According to a general algorithm, the $x$-range of the data set is divided into intervals separated by internal knots and adding end point knots for B-form spline construction. The number of coefficients is determined by the number of knots and the order of the spline polynomials of the sub-intervals. The weighted $\chi^{2}$ sum is minimized with respect to the coefficients of the base functions in the B-form. This will produce the best fit for fixed $\gamma$ with a minimized $\chi^{2}$ sum which will depend on $\gamma$. In the second step, we minimize the $\chi^{2}$ sum with respect to $\gamma$ to determine the best fit of the critical exponent. The one- $\sigma$ confidence interval is determined from the variation of the $\chi^{2}$ sum as a function of $\gamma$.

In Figure 3 we show three typical fits for illustration. The fit to the Goldstone pion in the PCAC channel improved as expected, with considerable increase in the error. The tension across channels decreased, as illustrated by comparison with the rho-channel fit, but the fit to $F_{\pi}$ remained unacceptable. With the extended data set we are unable to reproduce results in $[20,21]$ which used tables from our earlier limited subset of data [1] to argue in favor of consistency with the conformal phase. It is important to emphasize that we have not reached definitive conclusions about the failure of conformal tests. As we stated earlier [1], we have not analyzed yet the leading scaling violation effects and did not investigate if the good scaling form in separate quantum number channels can be explained in the chirally broken phase by strongly sqeezed wave function effects. In disagreement 
with [21], conformal FSS based analysis of the spectrum and related sum rules on moments of the correlators we have been developing are deep renormalization group based probes of the conformal phase. As explained in our forthcoming publication, we remain skeptical about the fitting procedure followed in [20] with efforts to rescue the conformal interpretation. The issues are not settled and ultimately will be decided in more definitive analyses.

\section{Acknowledgments}

We acknowledge support by the DOE under grant DE-FG02-90ER40546, by the NSF under grants 0704171 and 0970137, by the EU Framework Programme 7 grant (FP7/2007-2013)/ERC No 208740, and by the Deutsche Forschungsgemeinschaft grant SFB-TR 55. Computational resources were provided by USQCD at Fermilab and JLab, by the NSF XSEDE program, and by the University of Wuppertal. KH wishes to thank the Institute for Theoretical Physics and the Albert Einstein Center for Fundamental Physics at Bern University for their support. KH and JK wish to thank the Galileo Galilei Institute for Theoretical Physics and INFN for their hospitality and support at the workshop "New Frontiers in Lattice Gauge Theories".

\section{References}

[1] Z. Fodor, K. Holland, J. Kuti, D. Nogradi, C. Schroeder, Phys. Lett. B 703, 348 (2011).

[2] Z. Fodor, K. Holland, J. Kuti, D. Nogradi, C. Schroeder and C. H. Wong, PoS LAT2011, 073 (2011).

[3] Z. Fodor, K. Holland, J. Kuti, D. Nogradi, C. Schroeder and C. H. Wong, PoS LAT2012, 025 (2012).

[4] X. -Y. Jin and R. D. Mawhinney, PoS LATTICE 2011, 066 (2011) [arXiv:1203.5855 [hep-lat]].

[5] Y. Aoki et al. [LatKMI Collaboration], Phys. Rev. D 86, 054506 (2012).

[6] Y. Aoki et al. [LatKMI Collaboration], PoS LATTICE 2011, 053 (2011).

[7] D. Schaich, A. Cheng, A. Hasenfratz and G. Petropoulos, PoS LATTICE 2012, 028 (2012).

[8] A. Hasenfratz, A. Cheng, G. Petropoulos and D. Schaich, PoS LATTICE 2012, 034 (2012).

[9] A. Cheng, A. Hasenfratz and D. Schaich, Phys. Rev. D 85, 094509 (2012) [arXiv:1111.2317 [hep-lat]].

[10] A. Deuzeman, M. P. Lombardo, T. N. da Silva and E. Pallante, arXiv:1209.5720 [hep-lat].

[11] A. Deuzeman, M. P. Lombardo and E. Pallante, PoS LATTICE 2011, 083 (2011).

[12] A. Deuzeman, M. P. Lombardo, T. N. da Silva and E. Pallante, PoS LATTICE 2011, 321 (2011).

[13] K. Miura, M. P. Lombardo and E. Pallante, Phys. Lett. B 710, 676 (2012).

[14] Y. Aoki, Z. Fodor, S. D. Katz, K. K. Szabo, JHEP 0601, 089 (2006).

[15] L. Del Debbio, B. Lucini, A. Patella, C. Pica, A. Rago, Phys. Rev. D 82, 014510 (2010).

[16] F. Bursa, L. Del Debbio, L. Keegan, C. Pica, T. Pickup, Phys. Lett. B 696, 374-379 (2011).

[17] L. Del Debbio, R. Zwicky, Phys. Rev. D 82, 014502 (2010).

[18] L. Del Debbio and R. Zwicky, Phys. Lett. B 700, 217 (2011).

[19] L. Del Debbio, B. Lucini, A. Patella, C. Pica and A. Rago, arXiv:1111.4672 [hep-lat].

[20] T. Appelquist, G. T. Fleming, M. F. Lin, E. T. Neil and D. A. Schaich, Phys. Rev. D 84, 054501 (2011).

[21] T. DeGrand, Phys. Rev. D 84, 116901 (2011).

[22] C. de Boor, A Practical Guide to Splines, Springer-Verlag, 1978. 